\title{
FREQUENCY DOMAIN IDENTIFICATION OF THE HEAD-NECK COMPLEX
}

\author{
Mohammad Atapourfard, Tadashi Ishihara and Hikaru Inooka \\ Laboratory of Intelligent Control Systems. \\ Graduate School of Information Sciences. \\ Tohoku University. Sendai, Japan \\ Email: \{atapour, ishihara, inooka\} (a)control.is.tohoku.ac.jp
}

\begin{abstract}
The head-nech annplex (HNC) aesponses to the trumk horizontal (forr-and-aft) vilnation in the seated human body were studied It was assumed that the HNC has only rotational motion in mid-sagittal plane. An experimental method was resigned to generate the trunk horizontal vibration, and to measure the input and output signals to the system. The subjects were expesed to the raidon vibration at a magnitude of $1.60 \mathrm{~ms} \mathrm{~s}^{2}$ rms (root-mean-square) for 50 seconds. The coherenes and frequeney response function were then obtained on $0.5 \mathrm{~Hz}$ to $10 \mathrm{~Hz}$. The results manifested that the HNC behavior was quasi-linear with a dominant resenance frequency nearly between $1 \mathrm{~Hz}$ and $1.4 \mathrm{~Hz}$. Accordingly, a two-dimensional singleinverted-pendulum was considered as a model for the HNC. Frepueney domain identification method was then used to estimate the unknown parameters, in(ludeng the HXC viscoelastic and inertia paraneters, in the low fregueney range $(<3 \mathrm{~Hz})$. The moded was examined in the time domain using hoth random and sinusoidal vibration. Good agreement was obtained between experimental and simulation results.
\end{abstract}

\section{INTRODUCTION}

The dymanic responses of the head to the vertical and horizontal vibration have extensively been studied in the past three decades. The demands in automotive industry to develop the riding, quality of the passenger cars as well as other applications led many investigators to estimate the correlations between the head and seat vibration in the seated human body, or to model and identify the seated human body [1-5]. All these series of investigations have represented a set of fundamental data concerning the dynamic of entire body in the sitting posture. Nerertheless, the dymanic re sponses of the head-neck complex (HNC) to the trumk horizontal vibration have not been well investigated. Aceordingly, there may be less rexplicit knowledge regarding to the modeling and irlentification of the HNC:

Most of the attempts in the modeling and identification of the HNG concern the car collision analyses in which the head has been modeled by comsidering a lumped or distributed mass. And the flexilsility of the neck has been modeled by the rotational or lincar viscoelastic clements $[6]$. The incestigations show that the liead has both translational and rotational (flexion/extention) motion only in the high speed car collisions, while in the low speced collisions, the translational motion is negligible compared with the rotattional motion $[6,7]$. Furthermore, the motion of the head in the sat horizontal vibration |2| as woll as frontal or rear-end war collisions hate bern reported to occur in mid-sagittal plane (the median plane passes vertically through the middle of the body, dividing it equally into left and right halves).

The main objectives of this paper is to model and identify the HNC when it is exposed to the trunk horizontal vibration. It is assmed that the HNC has only rotational motions in mid-sagittal plane. Accordingly, we first present an experimental method to generate the trunk horizontal vibration, and to measure the input and output signals. Second, we investigate whether the input and the output signals to the HNC: are linearly correlated. Third, we present a simple model for the HNC, and we use the frequency domain identification method to identify the system. Finally, the viscoelastic parameters (spring and damping coef- 


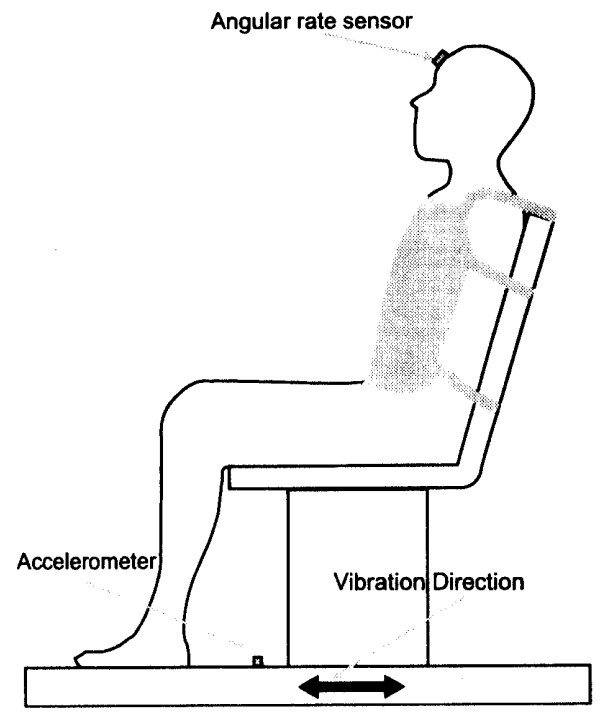

Fig. 1 The schematic draw of the experiment design

ficients) are estimated.

\section{METHOD}

\subsection{Experiments}

The experiments were conducted using an electrohydraulic vibrator. Its platform $(3 \mathrm{~m} \times 3 \mathrm{~m})$ had a stroke of $30 \mathrm{~cm}$, which had the capability of producing the signals from DC to $30 \mathrm{~Hz}$. A rigid seat was mounted on the platform. The flat (horizontal) surface of seat had a thin layer of cushion. The wooden seatback was adjustable in height to cover the posterior part of the trunk for each individual subject. It was inclined at an angle of 14 degrees to the vertical direction. The height of seat surface from the platform was $410 \mathrm{~mm}$ (Fig. 1).

Four male subjects (young and healthy) took part in this study. Their plysical characteristics are summarized in Table 1. Informed consent was obtained from each subject prior to the participation. None of subjects had any history of neck pain, diseases of cervical spine, neck injury, or musculoskeletal disorders. No subject was on any form of medication at the time of experiment. Each subject placed on the seat and the trunk of subject was then fixed to the seatback. This has been done using four straps, which were connected to a strong cloth covering anterior part of the trunk (Fig. 1). It was assumed that the horizontal vibration of the trunk was equal to that of the platform. During the experiment, the subjects were blindfolded and instructed to be relaxed and not to resist or apply any voluntary response by the neck muscles.

A surface-mounted accelerometer (Analog Devices
ADXL05) attached to the platform to measure the horizontal acceleration of the input to the HNC. An angular rate sensor (Nurata ENC-03J) was applied to measure the HNC angular velocity. This sensor was a lightweight type, pasted on the forehead of the subject to measure mainly in mid-sagittal plane, and to provide high-resolution measures of $\mathrm{HNC}$ angular velocity (Fig. 1). The input was a zero-mean Gaussian random vibration with $1.60 \mathrm{~ms}^{-2} \mathrm{rms}$ and a nominally flat spectrum. Each test run took 50 seconds. Each subject underwent this vibration four times individually, but for one of them, one more set of data was collected to use for validating the results. The measured signals, the platform acceleration and the angular velocity, were sampled at $100 \mathrm{~Hz}$ with an A/D converter and both band-pass filtered at 0.5 and $10 \mathrm{~Hz}$ with identical second order Butterworth filters.

The frequency response function (transfer function) of the system $H(f)$ can be derived, from experimental results, by using the cross-spectral density function method [8].

$$
H(f)=\frac{G_{x y}(f)}{G_{x x}(f)}
$$

where $G_{x y}(f)$ is cross spectrum of the input and the output, and $G_{x x}(f)$ is power spectrum of the input. The estimated transfer function using Eq. (1) only takes into account the linearly correlated proportion of the output with input. To delineate a single optimized transfer function for each subject, we have obtained an average value for the transfer function. The averaging method here was that of the geometric mean [9-11], which is defined by

$$
\overline{H(f)}=\prod_{k=1}^{n} \sqrt[n]{H_{k}(f)}
$$

where $H_{k}(f)$ indicates the complex form of the transfer function for the experiment number $k$, which is calculated from Eq. (1), and the value of $n$ indicates the number of experiments (here, $n=4$ ). It should be noted that the geometric mean of the transfer functions reduces the effects of noise-corruption and gives an unbiased estimation of transfer function better than that of the arithmetic mean $[9,10]$.

The results obtained by the use of cross-spectral density method should be qualified with additional approach. One method, to evaluate the quality of the estimated transfer function from the measured signals, is to determine the coherency function or ordinary coherency $C_{x y}(f)$ that is defined

$$
C_{x y}(f)=\frac{\left|G_{x y}(f)\right|}{G_{x x}(f) G_{y y}(f)} .
$$


Table 1 Physical characteristics of subjects (The \# sign indicates the subject number)

\begin{tabular}{|c|c|c|c|c|c|}
\hline Subject No & $\# 1$ & $\# 2$ & $\# 3$ & $\# 4$ & Mean (SD) \\
\hline Age $(\mathrm{yr})$ & 24 & 24 & 23 & 26 & $24.3(1.3)$ \\
\hline Height $(\mathrm{m})$ & 1.68 & 1.80 & 1.70 & 1.73 & $1.73(0.05)$ \\
\hline Weight $(\mathrm{kg})$ & 56 & 60 & 55 & 67 & $59.5(5.45)$ \\
\hline
\end{tabular}

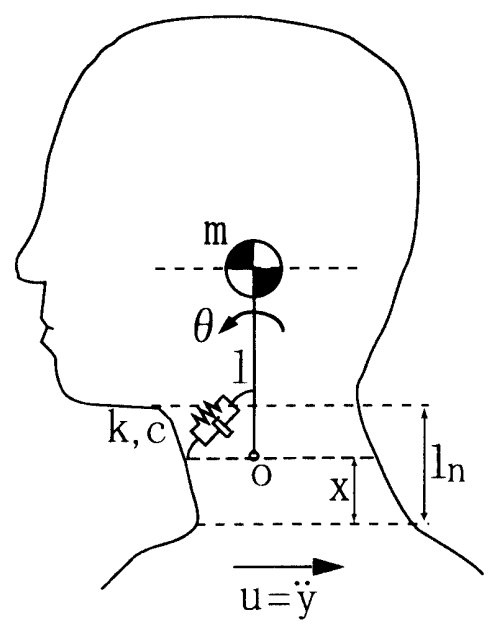

Fig. 2 A single-degree-of-freedom model of the HNC with its schematic draw

Coherence is a frequency dependant function that its value is always between 0 and unity. A value of coherence close to unity usually gives promise of a successful identification. If the system is linear and the input and output signals are not corrupted by noise, then the value of coherence will be unity. As the signal to noise ratio decreases, or behavior of the system tends to be nonlinear, the coherence also decreases [8]. If there is not any bidirectional relationship between input and output, then colerence will become o. Here for estimating both transfer function and coherency function, $0.1 \mathrm{~Hz}$ resolutions were considered.

\subsection{Modeling and Identification}

To model the system, the main simplifications were as follows. First, it was assumed that the motion of the $\mathrm{HNC}$ occurs only in mid-sagittal plane [2]. Second, the HNC only has flexion/extension motion, so that the translational motion of the $\mathrm{HNC}$ assumed to be negligible. Third, the HNC only has one center of rotation. A single-degree-of-freedom model (a twodimensional inverted pendulum) was then considered as a model for the HNC. This model was constituted of a torsional spring, a torsional damper and a lump mass (Fig. 2). The center of mass was assumed to be exactly over the center of rotation. The center of rotation was located at a distance $x$ from the joint of C7-T1 (Fig. 2). As a convention in this paper, the lower part of the neck that is shown by $x$ is called the lower neck and the other part of the neck that is located over the center of rotation is called the upper neck (Fig. 2). The linearized form of the equation of the system around $\theta=0$, was obtained in the following form

$$
I \ddot{\theta}+c \dot{\theta}+(k-m g l) \theta=m l \ddot{y}
$$

where $m$ is the mass of the head and the upper neck, and $I$ is the mass moment of inertia of the head and the upper neck, around the center of rotation o (Fig. 2). The $i f$ is the input (acceleration), the 7 is the distance between center of mass and center of rotation, $k$ indicates the spring factor, $c$ is the damping coefficient, and $\theta$ indicates the angular displacement of the system. The $m$ was then derived from

$$
m=m_{h}+m_{u n}
$$

where $m_{h}$ and $m_{u n}$ indicate mass of the head and the mass of upper neck, respectively. It has assumed that the neck had a constant length-density from the joint of $\mathrm{C} 0-\mathrm{C} 1$ to $\mathrm{C} 7-\mathrm{T} 1$ of the cervical vertebrae. Therefore, the mun was estimated by the following equation.

$$
m_{u n}=\left(\frac{l_{n}-x}{l_{n}}\right) m_{n}
$$

where $I_{n}$ is the length of the neck (from the joint of C0-C1 to C7-T1 of the (ervical vertebrae), and $m_{n}$ is the mass of the neck. It was assumed that the head and the neck center of mass were also exactly over the center of rotation. The distance between center of rotation and center of mass l (Fig. 2), was derived from

$$
I=\frac{m_{h}\left(l_{h}+l_{n}-x\right)+m_{n}\left(\frac{l_{n}-x}{2}\right)}{m}
$$

where $l_{h}$ indicates the distance between the head center of rotation and the $\mathrm{C} 0-\mathrm{C} 1$. The mass moment of incrtia of the upper neck $I_{u n}$ was estimated, from the Eq. (8), by assuming that the ratio of the masses and the ratio of radiuses-of-gyration of the upper neck and the neck were equal to the ratio of their lengths [13]. 
Table 2 Inertia parameters of the head and the neck

\begin{tabular}{|c|c|c|c|c|c|}
\hline Subject & $\# 1$ & $\# 2$ & $\# 3$ & $\# 4$ & Mean (SD) \\
\hline$m_{h}(\mathrm{~kg})$ & 4.10 & 4.55 & 4.38 & 4.80 & $4.46(0.29)$ \\
\hline$l_{h}(\mathrm{~cm})$ & 6.8 & 7.3 & 6.9 & 7.0 & $7.0(0.2)$ \\
\hline$I_{h}\left(\mathrm{~kg} \cdot \mathrm{m}^{2}\right)$ & 0.0201 & 0.0210 & 0.0199 & 0.0225 & $0.0209(0.0012)$ \\
\hline$m_{n}(\mathrm{~kg})$ & 1.11 & 1.19 & 1.09 & 1.33 & $1.18(0.11)$ \\
\hline$I_{n}(\mathrm{~cm})$ & 8.1 & 8.6 & 8.2 & 8.3 & $8.3(0.22)$ \\
\hline$I_{n}\left(\mathrm{~kg} \cdot \mathrm{m}^{2}\right)$ & 0.0013 & 0.0015 & 0.0013 & 0.0018 & $0.0015(0.0002)$ \\
\hline
\end{tabular}

$$
I_{u n}=\left(\frac{l_{n}-x}{l_{n}}\right)^{3} I_{n} .
$$

The I may be derived from the following equation.

$$
I=I_{h}+m_{n}\left(l_{n}+l_{n}-x\right)^{2}+I_{u n}+m_{u n}\left(\frac{l_{n}-x}{2}\right)^{2}
$$

The $m_{h}, l_{h}, I_{h}, m_{n}, l_{n}$, and $I_{n}$ were obtained according to the physical characteristics [13] of the subjects (Table 2). Therefore, the inertia parameters of the model, $m$ (Eq. (5)), $l$ (Eq. (7)), and $I$ (Eq. (9)) were found to be the functions of only one variable $x$.

The s-domain transfer function $H(s)$ of the system between the HNC angular velocity and input acceleration was obtained from Eq. (4).

$$
H(s)=e^{-s T_{d}} \frac{s}{\alpha s^{2}+\beta s+\gamma}
$$

where

$$
\begin{aligned}
& s=j 2 \pi f . \\
& a=\frac{I}{m !} . \\
& s=\frac{c}{m l} . \\
& \imath=\frac{k-m g l}{m l} .
\end{aligned}
$$

$T_{d}$ indicates the pure time delay of the system. The $\alpha, \beta, \gamma$, and $T_{d}$ were the unknown parameters in the Eq. (10). The pure time delay of system $T_{d}$ was estimated before the identification. The best value, which improved the quality of the identification, was found to be $40 \mathrm{~ms}$. Therefore, we had the three unknown parameters, $\alpha, \beta$, and $\gamma$ for identification.

Frequency domain identification method $[12,11]$ was used to identify the HNC. Therefore, the three unknown parameters, $\alpha, \beta$, and $\gamma$ (Eq. (10)) were estimated. Since the dominant resonance frequency of the system was found to be at low frequency range (see the result section) and the simplicity of the designed model was of great importance, we attempted to identify the system only at low frequency range $(<3 \mathrm{~Hz})$. The averaged frequency response function (Eq. (2)), corresponding to each subject, was used as a single experimental data for the identification [12]. In other words, it was attempted to fit the model to the averaged value of the experimental data. The variances of uncorrelated input and output noises were assumed to be Gaussian with zero mean value.

After obtaining the values of the $\alpha, \beta$, and $\gamma$ by the frequency domain identification, the following calculations were processed to get the final results. The variable $x$ was obtained from the Eq. (11). The vallues of the $m$ (Eq. (5)), l (Eq. (7)), and $I$ (Eq. (9)) were then derived. Finally, from the Eqs. (12 and 13), the spring factor $k$ and damping coefficient $c$ were obtained.

\section{RESULTS}

We found that, the frequency response of the $\mathrm{HNC}$ angular velocity to the trunk horizontal acceleration, corresponded to each of six subjects, had a dominant resonance frequency nearly between $1 \mathrm{~Hz}$ and $1.4 \mathrm{~Hz}$ (Fig. 3). The magnitudes of the transfer functions at the dominant resonance frequencies for subject number 1 to 6 were found to be $1.58,1.01,1.23,0.90,1.30$, and $1.53 \mathrm{rads}^{-1} / \mathrm{ms}^{-2}$, respectively. All subjects exhibited a second broad peak next to the resonance frequency of the system, which were extended up to nearly $7 \mathrm{~Hz}$. The patterns of the second broad peaks were slightly different for different subjects. However, in the second broad peaks, all subjects exhibited relatively greater magnitudes at around $6 \mathrm{~Hz}$.

The coherences revealed that the input and output of the $\mathrm{HNC}$ were well correlated, such that it was acceptable to use a linear model for the system. $79 \%$ of the mean values of the coherences corresponding to four subjects were found to be equal or greater than 0.8 (Fig. 4). For the frequency range of the $0.5 \mathrm{~Hz}$ to nearly $0.8 \mathrm{~Hz}$, the coherences showed relatively lower values with respect to the other frequency range $(0.8$ 


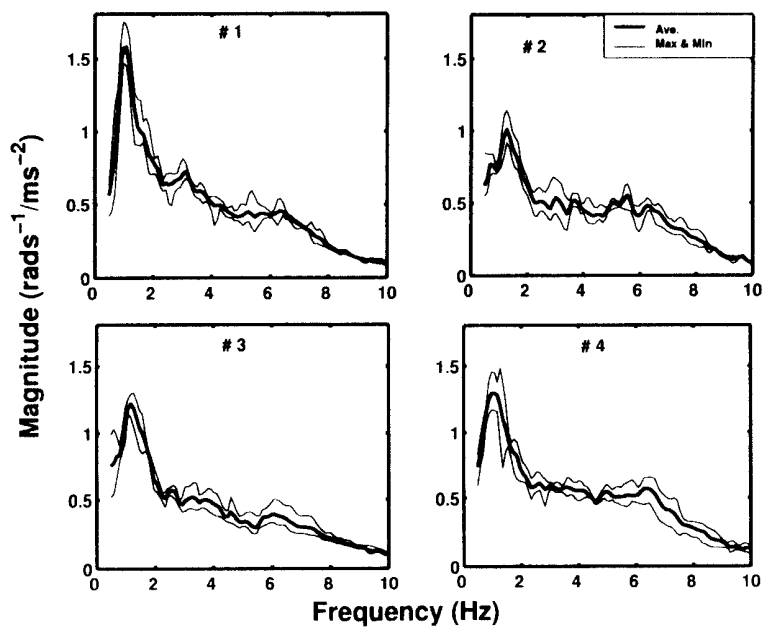

Fig. 3 The average (Ave.), maximum (Max.) and minimum (Min.) magnitudes of transfer functions that wore obtained from experimental data. Note that the \# sign indicates the subject number.

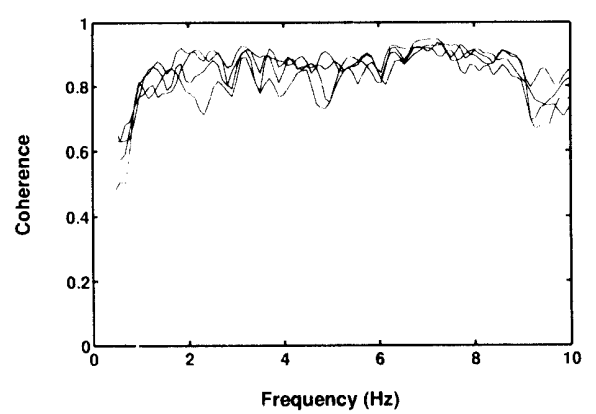

Fig. 4 The mean values of the coherency functions for four subjects. Each graph is related to one subject

$\mathrm{Hz}$ to $10 \mathrm{~Hz}$ ).

Ve determined the values of the unknown parameters corresponding to each of the subjects, including the parameters of the transfer functions $(\alpha, \beta$ and $\gamma)$, and inertia and viscoelastic parameters of the models (Table 3). We have also determined the normalized spring factor $k_{w}$, and damping coefficients $c_{w}$. Normalizations have been performed by dividing the values of $k$ and $c$ by the weight of the corresponding subject. The mean values of the parameters were derived by averaging the corresponding values for different subjects. The $k_{w}$ and $c_{w}$ exhibited less variances than the $k$ and ( between subjects. This may facilitate the future applications in which the viscoelastic parameters of the neck for new subjects are needed.

Frequency response function of the models demonstrated good agreements with geometric mean values of the experimentally derived frequency response func-

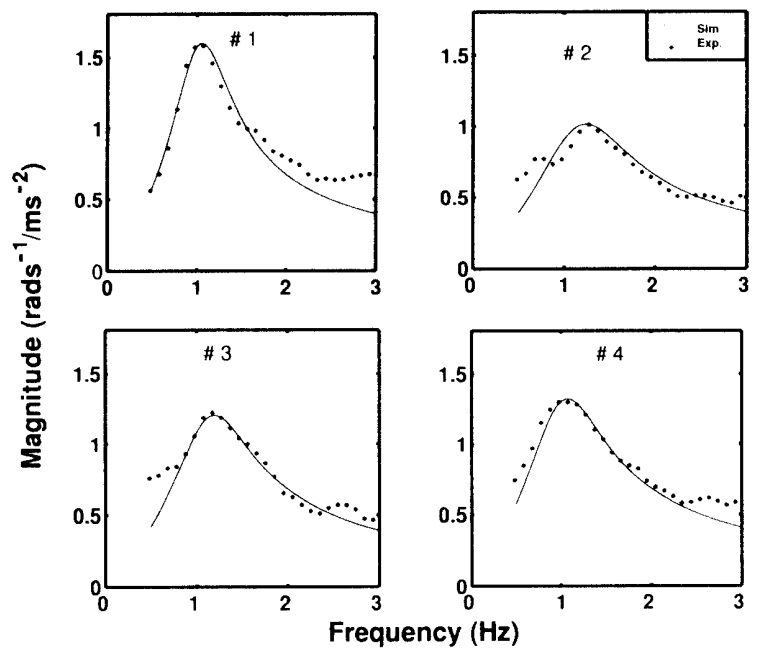

Fig. 5 Comparison of the experimental (Exp).) and simulated (Sim.) results of the magnitudes of the transfer functions for four subjects

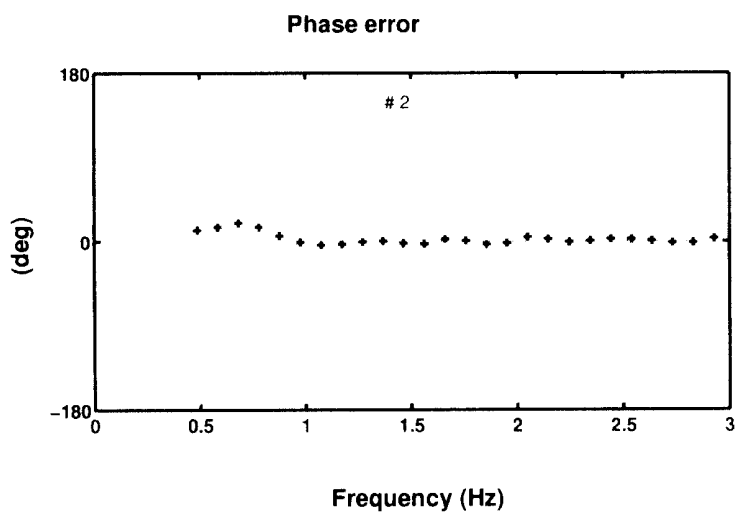

Fig. 6 Phase error between the model and experimental data for one subject

tions. Howerer, the results related to some of the sulbjects, e.g., subject number t, showed considerable discrepancy at frequencies lower than $0.8 \mathrm{~Hz}$ (Fig. 5). Wo have also shown the phase error between the model and experimental data for one subject (Fig. 6). This reveals that the phase of model has nearly same values as the system.

To validate the results, we have compared the simmlation and experimental results for one subject in the time domain. It was examined by independent random inputs, which were not used for the identification. In this case, the input and the output signals have been low-pass filtered $(<3 \mathrm{~Hz})$ with the identical filters. The output of the model was found to be nearly consistent with that of the measured data for both subjects in the frequencies lower than $3 \mathrm{~Hz}$ (Fig. $7)$. 
Table 3 The results of identification together with physical characteristics of four subjects

\begin{tabular}{|c|c|c|c|c|c|}
\hline Subject & $\# 1$ & $\# 2$ & $\# 3$ & $\# 4$ & Mean (SD) \\
\hline$\alpha$ & $\mathbf{0 . 1 4 8}$ & 0.149 & 0.151 & 0.141 & $0.147(0.004)$ \\
\hline$\beta$ & $\mathbf{0 . 6 2 7}$ & 0.987 & 0.830 & 0.754 & $0.800(0.151)$ \\
\hline$\gamma$ & $\mathbf{6 . 5 5 9}$ & 8.980 & 8.509 & 6.383 & $7.608(1.329)$ \\
\hline$x(\mathrm{~cm})$ & $\mathbf{3 . 5}$ & 4.4 & 3.2 & 5.4 & $4.1(1.0)$ \\
\hline$I(\mathrm{~cm})$ & $\mathbf{1 0 . 5}$ & 10.7 & 10.8 & 9.4 & $10.4(0.7)$ \\
\hline$I\left(\mathrm{kgm}^{2}\right)$ & $\mathbf{0 . 0 7 7 8}$ & 0.0820 & 0.0821 & 0.0699 & $0.0780(0.0057)$ \\
\hline$k(\mathrm{Nm} / \mathrm{rad})$ & $\mathbf{8 . 6 3}$ & 10.34 & 9.99 & 8.02 & $9.25(1.10)$ \\
\hline$c(\mathrm{Nms} / \mathrm{rad})$ & $\mathbf{0 . 3 3 1}$ & 0.534 & 0.453 & 0.374 & $0.423(0.090)$ \\
\hline$k_{w}(\mathrm{Nm} / \mathrm{kg}-\mathrm{rad})$ & $\mathbf{0 . 1 5 4}$ & 0.172 & 0.182 & 0.120 & $0.157(0.027)$ \\
\hline$c_{w}(\mathrm{Nms} / \mathrm{kg}-\mathrm{rad})$ & $\mathbf{0 . 0 0 5 9}$ & 0.0089 & 0.0082 & 0.0056 & $0.0072(0.0016)$ \\
\hline
\end{tabular}

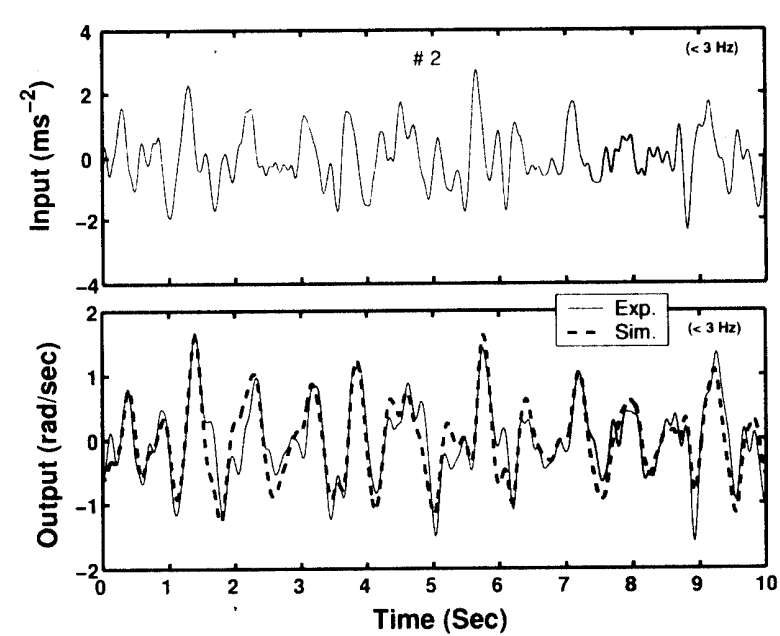

Fig. 7 Comparison of the experimental (Exp.) and simulated (Sim.) results when the input (upper panel) is a random vibration

\section{DISCUSSION}

The trunk horizontal vibration has been assumed to be equal to that of the seat vibration. This assumption has no considerable influence on the results of this research. Perhaps the coupling between the trunk and seatback influences the input signal like a low-pass filter with a cut-off frequency higher than $10 \mathrm{~Hz}$. Therefore, the only considerable effect, which it may have on the measurements, is to increase the pure time delay $\left(T_{d}\right)$ of the system. However, the pure time delay has no effect on the results of the identification.

We have observed a considerable discrepancy between the model and the measured result in the frequencies lower than $0.8 \mathrm{~Hz}$ (Fig. 5). In addition, the values of the coherences were found to be relatively low in this frequency range (Fig. 4). This was likely due to the active forces of the neck muscles that were included in the results of the measurements especially at low frequencies $(<0.8 \mathrm{~Hz})$. Accordingly, since the simulation of the active responses of the $\mathrm{HNC}$ is not the aim of our model, this observation may not reduce the validity of the model at even very low frequencies. The limitations of the excitation source that generated the input with lower signal to noise ratio $(\mathrm{S} / \mathrm{N})[8]$ may be the second cause of this discrepancy. Therefore, it may be concluded that our designed model represents the passive motion of the HNC at all frequencies lower than $3 \mathrm{~Hz}$.

We preferred to apply the frequency domain identification method to the time domain methods for identification of the HNC. As the system was not fully linear and the measured signals were corrupted by noises $[12$, 11], thus frequency domain identification method was more effective to characterize the system in appropriate frequency band, as well as to reduce the adverse effects of the noise on the identification, than the time domain methods. Since it was our aim to design a simple model with explicit results for the $\mathrm{HNC}$, we found that a second order model (single inverted pen(lulum) might be sufficient to represent the system in the frequency range less than $3 \mathrm{~Hz}$, where the dominant resonance frequency of the system existed. However, it should not be expected to find a simple linear model, or even a high-order model, which exactly has the same response as the system to the vibration since, physiologically and mechanically, the HNC is a complex system.

The choosing of the low frequency range $(<3 \mathrm{~Hz})$ for the identification and representing a simple model was due to the following main reasons: First, the dominant resonance frequency of the system was located at low frequencies. Second, for future applications of this research when it is intended to identify the active motion of the head, a simple model may be necessary, which can represent the passive motion of the head at low frequencies, as the neck muscles can be activated and response to the motion at frequencies lower than $2 \mathrm{~Hz}$ 
[14]. Since the model of this study is a simple model that represents the passive behavior of the system at low frequencies $(<3 \mathrm{~Hz})$, it is likely an appropriate model for such applications.

The viscoelastic parameters (spring and damping coefficients) of the HNC that were found in this study may also have future applications other than the objectives of this paper. For instance, in the low-velocity collision analyses, if it is needed to consider one center of rotation for the neck in the flexion/extension motion of the $\mathrm{HNC}$, then the mean values of the normalized viscoelastic parameters (Table 3 ) may be used by adjusting the values in accordance with the weight of the subject. Howerer, it should be noted that the results of this study were derived according to the measurements on four subjects. Therefore, study on more subjects (females and males) may lead to improve the accuracy of the results, and to facilitate the future applications.

\section{CONCLUSION}

The frequency response function of the $\mathrm{HNC}$ was obtained in the frequency range $0.5 \mathrm{~Hz}$ to $10 \mathrm{~Hz}$. It was found that the $\mathrm{HNC}$ in the trunk horizontal vibration had a dominant resonance frequency at around $1 \mathrm{~Hz}$ to $1.4 \mathrm{H} z$. Then, the system was modeled by the use of a two-dimensional single-inverted-pendulum in the frequency range lower than $3 \mathrm{~Hz}$. Frequency domain identification method successfully identified the $\mathrm{HNC}$ in the presence of the slight nonlinear distortions of the system, and the noise corruptions of the measured signals. The designed model was validated in the time domain. Finally, the inertia and viscoelastic parameters of the system were estimated.

\section{ACKNOWLEDGEMENTS}

The authors wish to express their thanks to Professor N. Inoue and Dr. N. Hori of the Department of Architecture and Building Science, Graduate School of Engineering. Tohoku University for their collaboration in conducting the experiments

\section{REFERERENCES}

[1] Griffin M. J.: Handbook of Human Vibration, Academic Press Ltd, Chap. 3 and 5 (1996).

[2] Paddan G. S., and Griffin M. J.: The Transmission of Translational Seat Vibration to the HeadII. Horizontal Vibration, J. Biomechanics. Vol. 21, No.3, pp.199-206 (1988)

[3] Paddan G. S., and Griffin M. J.: The Transmission of Translational Seat Vibration to the
Head-I. Vertical Seat Vibration J. Biomechanics, Vol.21, No.3, pp.191-197 (1988).

[4] Qassem W.: Model Prediction of Vibration Effects on Human Subject Seated on Various Cushions, Med. Eng. Phys., Vol.18, No.5, pp. 350-358 (1996).

[5] Muksian R., and D. Nash C.: A model for the Response of Seated Humans to Sinusoidal Displacements of the Seat J. Biomechanics. Vol.7, pp.209-215 (1974).

[6] Linder A.: A New Mathematical Neck Model for a Low-Velocity Rear-End Impact Dummy: Evaluation of Components Influencing Head Kinematics, Accid. Anal. and Prev.. Vol.32, pp.261$269(2000)$.

[7] Cholewicki J., Panjabi M. M., Nibu K., Babat L. B., Grauer J. N., and Drorak J.: Head Kinematics During In Vitro Whiplash Simulation, Acrid. Anal. and Pree. Vol.30, No.4, pp.469-479 (1998).

[8] Bendat J. S., and Piersol A. G.: Engineering Applications of Correlation and Spectral Analysis. Johan Wiley \& Sons (1980).

[9] Pintelon R., Schoukens J., and Renneboog J.: The Geometric Mean of Power (Amplitude) Spectra Has a Much Smaller Bias than the Classical Arithmetic: (RMS) Averaging, IEEE Transactions on Instrumentation and Measurement. Vol.37, No.2, pp.213-218 (1988).

[10] Schoukens J., and Pintelon R.: Measurement of Frequency Response Functions in Noisy Environments, IEEE Transactions on Instrumentation and Measurement. Vol.39, No.6, pp.905-909 (1990)

[11] Kollar I.: Frequency Domain System Identification Toolbox-User's Guide. The Mathworks, Inc (2001).

12] Pintelon R., and Schoukens .J.: System Identification. A Frequency Domain Approach. IEEE Press (2001).

[13] De Leva P.: Adjusments to ZatsiorskySeluyanov's Segment Incrtia Parameters, J. Biomechanics. Vol 29, no 9, pp. 1223-1230 (1996).

[14] Keshner E. A.: Modulating Active Stiffness Affects Head Stabilizing Strategies in Young and Elderly Adults During Trunk Rotations in the Vertical Plane, Gait and Posture. Vol.11, PP. $1-11(2000)$. 